\title{
Incomplete inverse spectral and nodal problems for Dirac operator
}

\author{
Zhaoying Wei ${ }^{*}$, Yongxia Guo ${ }^{2}$ and Guangsheng Wei
}

\section{"Correspondence:} imwzhy@163.com

${ }^{1}$ College of Science, Xi'an Shiyou University, Xi'an, 710065, P.R. China Full list of author information is available at the end of the article

\begin{abstract}
The incomplete inverse spectral and inverse nodal problems for Dirac operator defined on a finite interval with separated boundary conditions are considered. We prove uniqueness theorems for the so-called incomplete inverse spectral problem. Using the obtained result we show that for a unique determination of the operator it is sufficient to specify the nodal points only on a part of the interval slightly exceeding its half.
\end{abstract}

MSC: Primary 34L05; 34A55; secondary 34L40

Keywords: Dirac operator; inverse spectral problems; inverse nodal problems

\section{Introduction}

Consider the eigenvalue problem corresponding to the Dirac operator, denoted by $L:=$ $L(Q(x) ; \alpha, \beta)$, of the form

$$
l y:=B y^{\prime}-Q(x) y=\lambda y, \quad 0<x<1,
$$

with

$$
B=\left(\begin{array}{cc}
0 & 1 \\
-1 & 0
\end{array}\right), \quad Q(x)=\left(\begin{array}{cc}
p(x) & 0 \\
0 & q(x)
\end{array}\right), \quad y(x)=\left(\begin{array}{l}
y_{1}(x) \\
y_{2}(x)
\end{array}\right)
$$

subject to the boundary conditions

$$
\left\{\begin{array}{l}
U(y):=y_{1}(0) \cos \alpha+y_{2}(0) \sin \alpha=0, \\
V(y):=y_{1}(1) \cos \beta+y_{2}(1) \sin \beta=0,
\end{array} \quad 0 \leq \alpha, \beta<\pi .\right.
$$

Here $\lambda$ is a spectral parameter, $p(x), q(x) \in C^{1}[0,1]$ and they are real-valued functions. The Dirac operator is the relativistic Schrödinger operator in quantum physics. Here we mainly investigate inverse spectral and inverse nodal problems for the Dirac operator (1.1)-(1.2) and establish a connection between them.

The basic and comprehensive results about Dirac operators were given in [1]. Furthermore, spectral problems for Sturm-Liouville or Dirac operators were extensively studied in various publications; see e.g. [2-5]. Moreover, sampling theory is one of the most impor-

\section{Springer}

C 2015 Wei et al.; licensee Springer. This is an Open Access article distributed under the terms of the Creative Commons Attribution License (http://creativecommons.org/licenses/by/4.0), which permits unrestricted use, distribution, and reproduction in any medium, provided the original work is properly credited. 
tant mathematical tools used in communication engineering, the Whittaker-Kotel'nikovShannon (WKS) sampling theorem is viewed as the fundamental result in information theory [6-8]. In the past years, this sampling theorem has been investigated and improved by several authors. In particular, Tharwat and Bhrawy, etc. [9-14]) used the derivative sampling theorem (Hermite interpolations and sinc-method) to compute the eigenvalues of the discontinuous Dirac systems.

Inverse spectral problems consist in recovering operators from their spectral characteristics. Such problems play an important role in mathematics and have many applications in natural sciences and engineering (see $[2,5]$ and the references therein). Some aspects of inverse spectral problems for the Dirac systems were studied in $[1,15-18]$ and other papers. In particular, in [17] it is proved that under some conditions finitely many partially known spectra and partial information on the potential entirely determine the potential. Inverse nodal problems, in turn, consist in constructing operators from given nodes (zeros) of their eigenfunctions (refer to [19-24]). From the physical point of view this corresponds to finding, e.g., the density of a string or a beam from the zero-amplitude positions of the eigenvibrations. In [24] inverse nodal problems of reconstructing the Dirac operator on a finite interval were studied, where it was proved that the operator $L$ is determined uniquely by specifying a dense set of nodal points.

In the present paper, we will consider inverse problems of recovering $Q(x), \alpha, \beta$ from the given spectral and nodal characteristics. In what follows without loss of generality we always assume the mean value of $p(x)+q(x)$ is known a priori. Under this assumption we obtain uniqueness theorems and provide a constructive procedure for the solution. The novelty of this paper lies in the established connections between inverse nodal and spectral problems and the use of a set of nodal points of the components $y_{1}\left(x, \lambda_{n}\right)$ of the eigenfunctions $y\left(x, \lambda_{n}\right)=\left(y_{1}\left(x, \lambda_{n}\right), y_{2}\left(x, \lambda_{n}\right)\right)^{T}$ as the given nodal data. As far as we know, incomplete inverse nodal problem for the Dirac system had not been considered before and the obtained results are natural generalizations of the well-known results on the classical inverse problems.

The paper is organized as follows. In Section 2, we prove the uniqueness theorems for so-called incomplete inverse spectral problem for the Dirac operator $L$. Using the obtained result we show that for unique determination of the operator it is sufficient to specify the nodal points only on $(b, 1)$ with $b<1 / 2$ in Section 3 .

\section{Incomplete inverse spectral problem}

In the first part of the paper we study the so-called incomplete inverse problem of recovering the coefficients of (1.1)-(1.2) from a part of the spectrum of $L$ provided that they are known a priori on a part of the interval. We note that for recovering $Q(x)$ on the whole interval $[0,1]$ it is necessary to specify two spectra of boundary value problems with different boundary conditions (see [15]). We also note that the analogous problem for the Sturm-Liouville operators and differential pencils were investigated in [17, 25-27] and other work.

Let $S(x, \lambda), \varphi(x, \lambda)$ and $\psi(x, \lambda)$ be the solutions of Eq. (1.1) under the initial conditions

$$
S(0, \lambda)=\left(\begin{array}{l}
0 \\
1
\end{array}\right), \quad \varphi(0, \lambda)=\left(\begin{array}{c}
\sin \alpha \\
-\cos \alpha
\end{array}\right), \quad \psi(1, \lambda)=\left(\begin{array}{c}
\sin \beta \\
-\cos \beta
\end{array}\right)
$$


It is clear that, for each fixed $x \in[0,1]$, these solutions are entire in $\lambda$. Let $\tau=\operatorname{Im} \lambda$. Then the following representations hold uniformly in $x$ as $|\lambda| \rightarrow \infty$ (see [1], p.208, (5.11) and (5.12)):

$$
\left\{\begin{array}{l}
\varphi_{1}(x, \lambda)=\sin (\lambda x+\alpha+\eta(x))+\frac{V_{1}(x, \lambda)}{\lambda}+O\left(\frac{e(|| \mid x}{\lambda^{2}}\right), \\
\varphi_{2}(x, \lambda)=-\cos (\lambda x+\alpha+\eta(x))+\frac{V_{2}(x, \lambda)}{\lambda}+O\left(\frac{e|c| x}{\lambda^{2}}\right) .
\end{array}\right.
$$

Here

$$
\begin{aligned}
V_{1}(x, \lambda)= & \frac{(q-p)(x)}{4} \sin (\lambda x+\eta(x)+\alpha)+\frac{(q-p)(0)}{4} \sin (\lambda x+\eta(x)-\alpha) \\
& -\frac{\int_{0}^{x}(p(t)-q(t))^{2} \mathrm{~d} t}{8} \cos (\lambda x+\eta(x)+\alpha), \\
V_{2}(x, \lambda)= & \frac{(q-p)(x)}{4} \cos (\lambda x+\eta(x)+\alpha)+\frac{(p-q)(0)}{4} \cos (\lambda x+\eta(x)-\alpha) \\
& -\frac{\int_{0}^{x}(p(t)-q(t))^{2} \mathrm{~d} t}{8} \sin (\lambda x+\eta(x)+\alpha),
\end{aligned}
$$

and

$$
\eta(x)=\frac{1}{2} \int_{0}^{x}(p(t)+q(t)) \mathrm{d} t
$$

Denote $\Delta(\lambda):=\langle\psi(x, \lambda), \varphi(x, \lambda)\rangle$, where $\langle y(x), z(x)\rangle:=y_{1}(x) z_{2}(x)-y_{2}(x) z_{1}(x)$. The function $\Delta(\lambda)$ is called the characteristic function of $L$ and it does not depend on $x$. Substituting $x=0$ and $x=1$ into $\Delta(\lambda)$ we obtain $\Delta(\lambda)=V(\varphi)=-U(\psi)$. The function $\Delta(\lambda)$ is entire in $\lambda$ and its zeros $\left\{\lambda_{n}\right\}_{n \in \mathbb{Z}}$ coincide with the eigenvalues of $L$. It follows from (2.1) that for $|\lambda| \rightarrow \infty$

$$
\begin{aligned}
\Delta(\lambda)= & \cos \beta \varphi_{1}(1, \lambda)+\sin \beta \varphi_{2}(1, \lambda) \\
= & \sin (\lambda+\alpha-\beta+\eta(1))-\frac{\int_{0}^{1}(p(t)-q(t))^{2} \mathrm{~d} t}{8 \lambda} \cos (\lambda+\alpha-\beta+\eta(1)) \\
& +\frac{(q-p)(1)}{4 \lambda} \sin (\lambda+\alpha+\beta+\eta(1))+\frac{(q-p)(0)}{4 \lambda} \sin (\lambda-\alpha-\beta+\eta(1)) \\
& +O\left(\frac{e^{|\tau|}}{\lambda^{2}}\right),
\end{aligned}
$$

where $\eta(x)$ is defined in (2.2). It is well known that the spectrum of the problem (1.1)(1.2) consists of the eigenvalues $\lambda_{n}, n \in \mathbb{Z}$, which are all real and simple, and the sequence $\left\{\lambda_{n}, n \in \mathbb{Z}\right\}$ satisfies the classical asymptotic form [1]

$$
\lambda_{n}=n \pi+c_{0}+\frac{c_{1}}{n \pi}+O\left(\frac{1}{n^{2}}\right)
$$

where $c_{0}=\beta-\alpha-\eta(1)$ and

$$
c_{1}=\frac{1}{4}\left[(p-q)(1) \sin 2 \beta-(p-q)(0) \sin 2 \alpha+\frac{1}{2} \int_{0}^{1}(p-q)^{2} \mathrm{~d} t\right] .
$$


For our purpose of this paper, together with the problem $L$ defined by (1.1)-(1.2), we consider another problem $\tilde{L}$ of the same form but with different coefficients $\tilde{p}(x), \tilde{q}(x)$, $\tilde{\alpha}, \tilde{\beta}$. We agree that, everywhere below if a certain symbol $\delta$ denotes an object related to $L$, then $\tilde{\delta}$ will denote an analogous object related to $\tilde{L}$. Thus from the assumption we have $\eta(1)=\tilde{\eta}(1)$.

For the rest of the paper, we shall only consider the boundary condition $\alpha, \beta>0$. The other cases can be treated similarly.

Denote $e_{0}(x)=(\exp (2 i x), \exp (-2 i x))^{T}$. The following theorem has been proven by Horváth [28] for the Sturm-Liouville operator. We show it also holds for the Dirac operator (1.1)-(1.2).

Theorem 2.1 Fix $b \in(0,1 / 2]$. Let $\Lambda \subset \mathbb{Z}$ be a subset of integer numbers, and let $\Omega:=$ $\left\{\lambda_{n}\right\}_{n \in \Lambda}$ be a part of the spectrum of $L$ such that the system of functions $\left\{e_{0}\left(\lambda_{n} x\right)\right\}_{n \in \Lambda}$ is complete in $\left\{L_{2}(0, b)\right\}^{2}$. If $p(x)=\tilde{p}(x), q(x)=\tilde{q}(x)$ on $[b, 1]$, and $\beta=\tilde{\beta}, \Omega=\tilde{\Omega}, \eta(1)=\tilde{\eta}(1)$, then $p(x)=\tilde{p}(x), q(x)=\tilde{q}(x)$ on $[0,1]$ and $\alpha=\tilde{\alpha}$.

Remark 2.2 It is easy to see that if $b=1 / 2$ then Theorem 2.1 reduces to the Hochstadt and Lieberman's theorem [29] for the Dirac operator, which is studied by Malamud in [30]. At this point, our results are generalizations and improvements of the well-known results.

Proof Since $\Omega=\tilde{\Omega}$, it follows from (2.4) that $c_{0}=\tilde{c}_{0}$, which together with the conditions $\beta=\tilde{\beta}$ and $\eta(1)=\tilde{\eta}(1)$ yields $\alpha=\tilde{\alpha}$. Let us denote by $\varphi(x, \lambda)=\left(\varphi_{1}(x, \lambda), \varphi_{2}(x, \lambda)\right)^{T}$ the solution to the equation

$$
B y^{\prime}-Q(x) y=\lambda y
$$

with the initial condition

$$
\left(\begin{array}{l}
y_{1}(0, \lambda) \\
y_{2}(0, \lambda)
\end{array}\right)=\left(\begin{array}{c}
\sin \alpha \\
-\cos \alpha
\end{array}\right),
$$

and by $\tilde{\varphi}(x, \lambda)=\left(\tilde{\varphi}_{1}(x, \lambda), \tilde{\varphi}_{2}(x, \lambda)\right)^{T}$ the solution to the equation

$$
B \tilde{y}{ }^{\prime}-\tilde{Q}(x) \tilde{y}=\lambda \tilde{y}
$$

with the same initial condition as (2.6). Suppose $0 \leq a<b \leq \pi$, for all $\left(a_{1}, a_{2}\right)^{T}$ and $\left(b_{1}, b_{2}\right)^{T}$ in $\left(L_{2}[a, b]\right)^{2}$, define the inner product

$$
\left\langle\left(a_{1}, a_{2}\right)^{T},\left(b_{1}, b_{2}\right)^{T}\right\rangle=a_{1} b_{1}+a_{2} b_{2} .
$$

Multiplying $(2.5)$ by $\tilde{\varphi}(x, \lambda)$ and $(2.7)$ by $\varphi(x, \lambda)$ (in the sense of scalar product in $\mathbb{R}^{2}$ ), respectively, and subtracting, we get

$$
\frac{d}{d x}\left[\tilde{\varphi}_{1}(x, \lambda) \varphi_{2}(x, \lambda)-\tilde{\varphi}_{2}(x, \lambda) \varphi_{1}(x, \lambda)\right]+\langle(\tilde{Q}(x)-Q(x)) \varphi(x, \lambda), \tilde{\varphi}(x, \lambda)\rangle=0 .
$$


Integrating the above equality from 0 to 1 with respect to $x$, we infer from the conditions $p(x)=\tilde{p}(x), q(x)=\tilde{q}(x)$ on $[b, 1]$ and $\alpha=\tilde{\alpha}$ that

$$
\begin{aligned}
\int_{0}^{b}\langle(Q(t)-\tilde{Q}(t)) \varphi(t, \lambda), \tilde{\varphi}(t, \lambda)\rangle \mathrm{d} t & =\tilde{\varphi}_{1}(1, \lambda) \varphi_{2}(1, \lambda)-\tilde{\varphi}_{2}(1, \lambda) \varphi_{1}(1, \lambda) \\
& =\left[\tilde{\varphi}_{1}(1, \lambda) \Delta(\lambda)-\tilde{\Delta}(\lambda) \varphi_{1}(1, \lambda)\right] / \sin \beta .
\end{aligned}
$$

Define

$$
p_{1}(x)=p(x)-\tilde{p}(x), \quad q_{1}(x)=q(x)-\tilde{q}(x)
$$

and

$$
H(\lambda)=\int_{0}^{b}\langle(Q(t)-\tilde{Q}(t)) \varphi(t, \lambda), \tilde{\varphi}(t, \lambda)\rangle \mathrm{d} t
$$

Since $\Delta\left(\lambda_{n}\right)=\tilde{\Delta}\left(\lambda_{n}\right)=0$ for $n \in \Lambda$, it follows from (2.8) that

$$
H\left(\lambda_{n}\right)=0, \quad n \in \Lambda
$$

It is known [1] that there exist kernels $K(x, t)=\left(K_{i j}(x, t)\right)_{i, j=1}^{2}$ and $\tilde{K}(x, t)=\left(\tilde{K}_{i j}(x, t)\right)_{i, j=1}^{2}$ with entries continuously differentiable on $0 \leq t \leq x \leq 1$ such that

$$
\left\{\begin{array}{l}
\varphi(x, \lambda)=\varphi_{0}(x, \lambda)+\int_{0}^{x} K(x, t) \varphi_{0}(t, \lambda) \mathrm{d} t, \\
\tilde{\varphi}(x, \lambda)=\varphi_{0}(x, \lambda)+\int_{0}^{x} \tilde{K}(x, t) \varphi_{0}(t, \lambda) \mathrm{d} t,
\end{array}\right.
$$

where $\varphi_{0}(x, \lambda)=(\sin (\lambda x+\alpha),-\cos (\lambda x+\alpha))^{T}$. Thus we can show from (2.10) that

$$
\begin{aligned}
H(\lambda)= & \int_{0}^{b}\langle(Q(t)-\tilde{Q}(t)) \varphi(t, \lambda), \tilde{\varphi}(t, \lambda)\rangle \mathrm{d} t \\
= & \int_{0}^{b} p_{1}(t)\left[-\cos 2(\lambda t+\alpha)+\int_{0}^{t} R_{1}(t, s) e^{2 i \lambda s} \mathrm{~d} s+\int_{0}^{t} R_{2}(t, s) e^{-2 i \lambda s} \mathrm{~d} s\right] \mathrm{d} t \\
& +\int_{0}^{b} q_{1}(t)\left[-\sin 2(\lambda t+\alpha)+\int_{0}^{t} R_{3}(t, s) e^{2 i \lambda s} \mathrm{~d} s+\int_{0}^{t} R_{4}(t, s) e^{-2 i \lambda s} \mathrm{~d} s\right] \mathrm{d} t \\
= & \int_{0}^{b} f_{1}(t)\left[e^{2 i \lambda t}+\int_{0}^{t} S_{11}(t, s) e^{2 i \lambda s} \mathrm{~d} s+\int_{0}^{t} S_{12}(t, s) e^{-2 i \lambda s} \mathrm{~d} s\right] \mathrm{d} t \\
& +\int_{0}^{b} f_{2}(t)\left[e^{-2 i \lambda t}+\int_{0}^{t} S_{21}(t, s) e^{2 i \lambda s} \mathrm{~d} s+\int_{0}^{t} S_{22}(t, s) e^{-2 i \lambda s} \mathrm{~d} s\right] \mathrm{d} t \\
= & \int_{0}^{b} e^{2 i \lambda s}\left[f_{1}(s)+\int_{s}^{b} f_{1}(t) S_{11}(t, s)+f_{2}(t) S_{21}(t, s) \mathrm{d} t\right] \mathrm{d} s \\
& +\int_{0}^{b} e^{-2 i \lambda s}\left[f_{2}(s)+\int_{s}^{b} f_{1}(t) S_{12}(t, s)+f_{2}(t) S_{22}(t, s) \mathrm{d} t\right] \mathrm{d} s \\
= & \int_{0}^{b}\left\langle e_{0}(\lambda s), f(s)+\int_{s}^{b} S(t, s) f(t) \mathrm{d} t\right\rangle \mathrm{d} s,
\end{aligned}
$$


where

$$
f(x)=\left(f_{1}(x), f_{2}(x)\right)^{T}=\left(-\frac{e^{2 i \alpha}}{2 i}\left(q_{1}(x)+i p_{1}(x)\right), \frac{e^{-2 i \alpha}}{2 i}\left(q_{1}(x)-i p_{1}(x)\right)\right)^{T},
$$

and $R_{l}(x, t), l=1, \ldots, 4, S(x, t)=\left(S_{i j}(x, t)\right), i, j=1,2$ are matrix functions with entries which are piecewise-continuously differentiable on $0 \leq t \leq x \leq 1$. Taking (2.9) into account, it yields

$$
0=H\left(\lambda_{n}\right)=\int_{0}^{b}\left\langle e_{0}\left(\lambda_{n} s\right), f(s)+\int_{s}^{b} S(t, s) f(t) \mathrm{d} t\right\rangle \mathrm{d} s, \quad n \in \Lambda .
$$

Thus from the completeness of the functions $\left\{e_{0}\left(\lambda_{n} x\right)\right\}_{n \in \Lambda}$ in $\left\{L_{2}(0, b)\right\}^{2}$, it follows that

$$
f(s)+\int_{s}^{b} S(t, s) f(t) \mathrm{d} t=0 \quad \text { for } s \in(0, b) .
$$

But this equation is a homogeneous Volterra integral equation and has only the zero solution. Thus we have obtained $f(x)=\left(f_{1}(x), f_{2}(x)\right)^{T}=0$ on $[0, b]$ and consequently $p_{1}(x)=$ $q_{1}(x)=0$, i.e. $p(x)=\tilde{p}(x), q(x)=\tilde{q}(x)$ on $[0, b]$. This completes the proof.

In Section 3 the following theorem will be used.

Theorem 2.3 Fix $b<1 / 2$ and $N \in \mathbb{N}$. Let $\beta=\tilde{\beta}, p(x)=\tilde{p}(x), q(x)=\tilde{q}(x)$ on $[b, 1]$. If $\left\{\lambda_{n}\right\}_{|n| \geq N}=\left\{\tilde{\lambda}_{n}\right\}_{|n| \geq N}$ and $\eta(1)=\tilde{\eta}(1)$, then $L=\tilde{L}$, i.e., $p(x)=\tilde{p}(x), q(x)=\tilde{q}(x)$ on $[0,1]$ and $\alpha=\tilde{\alpha}$.

Proof It follows from the conditions of the theorem that

$$
\alpha=\tilde{\alpha}, \quad \eta(a)=\tilde{\eta}(a) .
$$

Let $\Phi(x, \lambda)$ and $\Psi(x, \lambda)$ be the solutions of Eq. (1.1) under the boundary conditions

$$
U(\Phi)=1, \quad V(\Phi)=0 ; \quad \Psi_{1}(0, \lambda)=1, \quad V(\Psi)=0 .
$$

Then a direct calculation yields

$$
\left\{\begin{array}{l}
\Phi(x, \lambda)=-\frac{\psi(x, \lambda)}{\Delta(\lambda)}=\frac{1}{\sin \alpha}[S(x, \lambda)+M(\lambda) \varphi(x, \lambda)], \\
\Psi(x, \lambda)=\frac{\psi(x, \lambda)}{\psi_{1}(0, \lambda)}=\frac{1}{\sin \alpha}\left[\varphi(x, \lambda)+M_{0}(\lambda) S(x, \lambda)\right],
\end{array}\right.
$$

where

$$
M(\lambda)=-\frac{\psi_{1}(0, \lambda)}{\Delta(\lambda)} \quad \text { and } \quad M_{0}(\lambda)=-\frac{\Delta(\lambda)}{\psi_{1}(0, \lambda)}=\frac{1}{M(\lambda)} .
$$

Denote

$$
D(\lambda)=\left.\langle\varphi(x, \lambda), \tilde{\varphi}(x, \lambda)\rangle\right|_{x=b} .
$$


With the help of (2.12)-(2.13), we calculate for all $|n| \geq N$

$$
\left.\frac{D(\lambda)}{\sin \alpha}\right|_{\lambda=\lambda_{n}}=\left\langle\Psi(b, \lambda), \tilde{\Psi}(b, \lambda)||_{\lambda=\lambda_{n}}=\left.\frac{\langle\psi(b, \lambda), \tilde{\psi}(b, \lambda)\rangle}{\psi_{1}(0, \lambda) \tilde{\psi}_{1}(0, \lambda)}\right|_{\lambda=\lambda_{n}} .\right.
$$

Under the conditions of the theorem we have $\psi(x, \lambda)=\tilde{\psi}(x, \lambda)$ for $x \in[b, 1]$, hence

$$
D\left(\lambda_{n}\right)=0, \quad|n| \geq N
$$

Moreover, we can infer from (2.1), (2.11) and (2.14) that

$$
D(\lambda)=O\left(\frac{e^{2|\tau| a}}{\lambda}\right) .
$$

Consider the function

$$
F(\lambda)=\frac{D(\lambda)}{\Delta(\lambda)}\left(\lambda-\lambda_{0}\right) \prod_{n=1}^{N-1}\left(\lambda-\lambda_{-n}\right)\left(\lambda-\lambda_{n}\right)
$$

which by virtue of (2.15) is entire in $\lambda$. On the other hand, according to (2.3) and with the help of [31], pp.118-119, we have for sufficiently large $|\lambda|$

$$
|\Delta(\lambda)| \geq C_{\delta} e^{|\tau|}, \quad \lambda \in G_{\delta}
$$

where $G_{\delta}=\left\{\lambda:\left|\lambda-n \pi-c_{0}\right| \geq \delta, n \in \mathbb{Z}\right\}$, which together with (2.16) implies that

$$
F(\lambda)=O\left(\lambda^{2 N-2} \exp ((2 a-1)|\tau|)\right), \quad|\lambda| \rightarrow \infty, \lambda \in G_{\delta} .
$$

Using Phragmen-Lindelöf and Liouville's theorems we arrive at $F(\lambda) \equiv 0$. Consequently, $D(\lambda) \equiv 0$, which means

$$
m_{-}(b, \lambda)=-\frac{\varphi_{2}(b, \lambda)}{\varphi_{1}(b, \lambda)}=-\frac{\tilde{\varphi}_{2}(b, \lambda)}{\tilde{\varphi}_{1}(b, \lambda)}=\tilde{m}_{-}(b, \lambda) .
$$

The result is obtained immediately from the uniqueness theorem in [17] and this completes the proof.

\section{Incomplete inverse nodal problem}

In the first part of this section we obtain uniqueness theorem of recovering the potential $Q(x)$ on the whole interval $[0,1]$ and the parameters $\alpha, \beta$ in the boundary conditions from a dense subset of nodal points. Further, using the obtained result in Section 2 and developing the idea of the works $[25,27]$ we prove that for a unique determination of $L$ it is sufficient to specify the set of nodal points only on $[b, 1]$ with $b<1 / 2$.

First we study the oscillation property of the first component $y_{1}\left(x, \lambda_{n}\right)$ of the eigenfunction $y\left(x, \lambda_{n}\right)$ of the Dirac system for sufficiently large $|n|$. 
Lemma 3.1 For sufficiently large $|n|$, the first component $y_{1}\left(x, \lambda_{n}\right)$ of the eigenfunction $y\left(x, \lambda_{n}\right)$ of the Dirac system has exactly $|n|$ nodes in the interval $(0,1)$ :

$$
\begin{aligned}
& 0<x_{n}^{1}<\cdots<x_{n}^{n}<1 \text { for } n>0, \\
& 0<x_{n}^{0}<\cdots<x_{n}^{n+1}<1 \quad \text { for } n<0 .
\end{aligned}
$$

Moreover,

$$
\begin{aligned}
x_{n}^{j}= & \frac{j \pi-\alpha-\eta\left(x_{n}^{j}\right)}{n \pi}-\frac{c_{0}\left(j \pi-\alpha-\eta\left(x_{n}^{j}\right)\right)-c_{2}}{(n \pi)^{2}} \\
& +\frac{\left(c_{0}^{2}-c_{1}\right)\left(j \pi-\alpha-\eta\left(x_{n}^{j}\right)\right)}{(n \pi)^{3}}+O\left(\frac{1}{n^{3}}\right)
\end{aligned}
$$

uniformly with respect to $j \in \mathbb{Z}$, where

$$
c_{2}=\frac{\sin 2 \alpha}{4}(q-p)(0)+\frac{1}{8} \int_{0}^{x_{n}^{\prime}}(p(t)-q(t))^{2} \mathrm{~d} t .
$$

Proof We note that the eigenfunctions $\left(y_{1}\left(x, \lambda_{n}\right), y_{2}\left(x, \lambda_{n}\right)\right)^{T}$ of the Dirac operator $L$ are real-valued. From (2.1) we see that the function $y_{1}\left(x, \lambda_{n}\right)$ has the following asymptotic formula for sufficiently large $|n|$, uniformly in $x$ :

$$
\begin{aligned}
y_{1}\left(x, \lambda_{n}\right)= & \sin \left(\lambda_{n} x+\alpha+\eta(x)\right)-\frac{\int_{0}^{x}(p(t)-q(t))^{2} \mathrm{~d} t}{8 \lambda_{n}} \cos \left(\lambda_{n} x+\alpha+\eta(x)\right) \\
& +\frac{(q-p)(x)}{4 \lambda_{n}} \sin \left(\lambda_{n} x+\alpha+\eta(x)\right)+\frac{(q-p)(0)}{4 \lambda_{n}} \sin \left(\lambda_{n} x-\alpha+\eta(x)\right)+O\left(\frac{1}{\lambda_{n}^{2}}\right) .
\end{aligned}
$$

From $y_{1}\left(x_{n}^{j}, \lambda_{n}\right)=0$, we get

$$
\tan \left(\lambda_{n} x_{n}^{j}+\alpha+\eta\left(x_{n}^{j}\right)\right)=\frac{\int_{0}^{x_{n}^{j}}(p-q)^{2} \mathrm{~d} t+2 \sin 2 \alpha(q-p)(0)}{8 \lambda_{n}}+O\left(\frac{1}{\lambda_{n}^{2}}\right) .
$$

Using Taylor's expansion for the arctangent, we have

$$
x_{n}^{j}=\frac{1}{\lambda_{n}}\left[j \pi-\alpha-\eta\left(x_{n}^{j}\right)\right]+\frac{c_{2}}{\lambda_{n}^{2}}+O\left(\frac{1}{\lambda_{n}^{3}}\right),
$$

where $c_{2}$ is defined in (3.3). Furthermore, using the asymptotic formula

$$
\lambda_{n}^{-1}=\frac{1}{n \pi}-\frac{c_{0}}{(n \pi)^{2}}+\frac{c_{0}^{2}-c_{1}}{(n \pi)^{3}}+O\left(\frac{1}{n^{4}}\right)
$$

we conclude that equality (3.2) holds.

Equality (3.2) gives the asymptotic expansion for the nodal lengths $l_{n}^{j}:=x_{n}^{j+1}-x_{n}^{j}$; we have uniformly with respect to $j$

$$
\begin{aligned}
l_{n}^{j} & =\frac{\pi-\frac{1}{2} \int_{x_{n}^{j}}^{x_{n}^{j+1}}(p(t)+q(t)) \mathrm{d} t}{n \pi}-\frac{c_{0}\left(\pi-\frac{1}{2} \int_{x_{n}^{j}}^{x_{n}^{j+1}}(p(t)+q(t)) \mathrm{d} t\right)}{(n \pi)^{2}}+O\left(\frac{1}{n^{3}}\right) \\
& =\frac{1}{n}+o\left(\frac{1}{n}\right), \quad|n| \rightarrow \infty .
\end{aligned}
$$


Consequently, for large $|n|$ we have $x_{n}^{j}<x_{n}^{j+1}$ for positive $n$ and $x_{n}^{j}>x_{n}^{j+1}$ for negative $n$. The asymptotic formula (3.2) gives for $j=0, \pm 1, n, n+1$ that

$$
\begin{aligned}
& x_{n}^{-1}=\frac{-\pi-\alpha}{n \pi}+O\left(\frac{1}{n^{2}}\right), \quad x_{n}^{0}=\frac{-\alpha}{n \pi}+O\left(\frac{1}{n^{2}}\right), \\
& x_{n}^{1}=\frac{\pi-\alpha}{n \pi}+O\left(\frac{1}{n^{2}}\right), \quad \ldots, \quad x_{n}^{n}=1-\frac{\alpha}{n \pi}+O\left(\frac{1}{n^{2}}\right), \\
& x_{n}^{n+1}=1+\frac{\pi-\alpha}{n \pi}+O\left(\frac{1}{n^{2}}\right) .
\end{aligned}
$$

Thus, according to the order of $x_{n}^{j}$, for large $|n|$, the first component $y_{1}\left(x, \lambda_{n}\right)$ of the eigenfunction $y\left(x, \lambda_{n}\right)$ of the Dirac system has exactly $|n|$ nodes in the interval $(0,1)$, i.e., $x_{n}^{j}$, $j=\overline{1, n}$ for positive $n$ and $x_{n}^{j}, j=\overline{n+1,0}$ for negative $n$. The proof is complete.

Corollary 3.2 From Lemma 3.1 and (3.4) it follows that the sets $X_{1}=\left\{x_{n}^{j}\right\}_{n>0}, X_{2}=\left\{x_{n}^{j}\right\}_{n<0}$ and $X_{0}=X_{1} \cup X_{2}$ are all dense in $(0,1)$.

Definition 3.3 Let $X \subset X_{j}, j=0,1,2$. The set $X$ is called twin if together with each of its points $x_{n}^{j}$ the set $X$ contains at least one of adjacent nodal points $x_{n}^{j-1}$ and/or $x_{n}^{j+1}$.

Let us now formulate a uniqueness theorem and provide a constructive procedure for the solution of the inverse nodal problem.

Theorem 3.4 Fix $i=1,2$. Let $X \subset X_{i}$ be a subset nodal points which is dense and twin in $(0,1)$. If $X=\tilde{X}$ and $\eta(1)=\tilde{\eta}(1)$, then $p(x)=\tilde{p}(x), q(x)=\tilde{q}(x)$ on $[0,1]$ and $\alpha=\tilde{\alpha}, \beta=\tilde{\beta}$.

Remark 3.5 Note that in this theorem the dense subset $X$ only need to be known for $n>0$ or $n<0$ rather than both, i.e., the known subset $X$ do not need to be contained in $X_{0}$. Similar results can be obtained using a dense subset of nodal points of the second component $y_{2}\left(x, \lambda_{n}\right)$ as the given spectral data for recovering the Dirac system.

Proof of Theorem 3.4 We first prove the given nodal set $X$ uniquely determines the parameters $\alpha, \beta$ and the function $p(x)+q(x)$ on $[0,1]$. For each fixed $x \in[0,1]$ choose a sequence $\left\{x_{n}^{j}\right\} \subset X_{i}$ such that $x_{n}^{j} \rightarrow x$ as $|n| \rightarrow \infty$. Using the asymptotic expansion (3.2), we get

$$
n \pi x_{n}^{j}-j \pi=-\alpha-\eta\left(x_{n}^{j}\right)-\frac{j}{n} c_{0}+O\left(\frac{1}{n}\right) .
$$

Also the fact $\lim _{|n| \rightarrow \infty} x_{n}^{j}=x$ implies that $j / n \rightarrow x$ and $\eta\left(x_{n}^{j}\right) \rightarrow \eta(x)$. From this it follows that as $|n| \rightarrow \infty$ the limit of the left-hand side of (3.5) exists and

$$
g_{i}(x):=\lim _{|n| \rightarrow \infty}\left[n \pi x_{n}^{j}-j \pi\right]=-\alpha-\eta(x)-c_{0} x .
$$

Taking the values for $g_{i}(x)$ at $x=0$ and $x=1$, respectively, we obtain

$$
\alpha=-g_{i}(0), \quad \beta=-g_{i}(1) .
$$


After $\alpha$ and $\beta$ are reconstructed, taking derivatives of the function $g_{i}(x)$, we derive

$$
p(x)+q(x)-\int_{0}^{1}(p(t)+q(t)) \mathrm{d} t=-2\left[g_{i}^{\prime}(x)-\alpha+\beta\right] .
$$

Note that if $X=\tilde{X}$, then (3.6) yields $g_{i}(x) \equiv \tilde{g}_{i}(x), x \in[0,1]$. By virtue of (3.7)-(3.8) and the assumption $\eta(1)=\tilde{\eta}(1)$, we get

$$
\alpha=\tilde{\alpha}, \quad \beta=\tilde{\beta}, \quad p(x)+q(x)=\tilde{p}(x)+\tilde{q}(x) \quad \text { on }[0,1] .
$$

Next we are going to show $p(x)=\tilde{p}(x), q(x)=\tilde{q}(x)$ on $[0,1]$. Multiply (2.5) with $\lambda=\lambda_{n}$ by $\tilde{\varphi}\left(x, \tilde{\lambda}_{n}\right)$ and (2.7) with $\lambda=\tilde{\lambda}_{n}$ by $\varphi\left(x, \lambda_{n}\right)$ (in the sense of scalar product in $\left.\mathbb{R}^{2}\right)$, respectively, subtract the two equations and integrate it from $x_{n}^{j}$ to $x_{n}^{j+1}$. This result is

$$
\begin{aligned}
0= & \int_{x_{n}^{j}}^{x_{n}^{j+1}}\left\langle\left(\tilde{Q}(t)-Q(t)-\left(\lambda_{n}-\tilde{\lambda}_{n}\right) I\right) \varphi\left(t, \lambda_{n}\right), \tilde{\varphi}\left(t, \tilde{\lambda}_{n}\right)\right\rangle \mathrm{d} t \\
= & \int_{x_{n}^{j}}^{x_{n}^{j+1}}(\tilde{p}(t)-p(t)) \varphi_{1}\left(t, \lambda_{n}\right) \tilde{\varphi}_{1}\left(t, \tilde{\lambda}_{n}\right) \mathrm{d} t+\int_{x_{n}^{j}}^{x_{n}^{j+1}}(\tilde{q}(t)-q(t)) \varphi_{2}\left(t, \lambda_{n}\right) \tilde{\varphi}_{2}\left(t, \tilde{\lambda}_{n}\right) \mathrm{d} t \\
& +\left(\tilde{\lambda}_{n}-\lambda_{n}\right) \int_{x_{n}^{j}}^{x_{n}^{j+1}}\left[\tilde{\varphi}_{1}\left(t, \tilde{\lambda}_{n}\right) \varphi_{1}\left(t, \lambda_{n}\right)+\varphi_{2}\left(t, \lambda_{n}\right) \tilde{\varphi}_{2}\left(t, \tilde{\lambda}_{n}\right)\right] \mathrm{d} t .
\end{aligned}
$$

Since $\eta(1)=\tilde{\eta}(1)$, it yields from (2.1), (2.4) and (3.9)

$$
\lambda_{n}-\tilde{\lambda}_{n}=O\left(\frac{1}{n}\right)
$$

and

$$
\left\{\begin{array}{l}
\tilde{y}_{1}\left(x, \tilde{\lambda}_{n}\right) y_{1}\left(x, \lambda_{n}\right)=\frac{1}{2}\left[1-\cos \left(\left(\lambda_{n}+\tilde{\lambda}_{n}\right) x+2(\alpha+\eta(x))\right)\right]+O\left(\frac{1}{n}\right) \\
\tilde{y}_{2}\left(x, \tilde{\lambda}_{n}\right) y_{2}\left(x, \lambda_{n}\right)=\frac{1}{2}\left[1+\cos \left(\left(\lambda_{n}+\tilde{\lambda}_{n}\right) x+2(\alpha+\eta(x))\right)\right]+O\left(\frac{1}{n}\right) .
\end{array}\right.
$$

From the above equalities we get from (3.10) and (3.11)

$$
\begin{aligned}
0= & \int_{x_{n}^{j}}^{x_{n}^{j+1}}[(\tilde{q}(t)-q(t))+(\tilde{p}(t)-p(t))] \mathrm{d} t \\
& +\int_{x_{n}^{j}}^{x_{n}^{j+1}}[(\tilde{q}(t)-q(t))-(\tilde{p}(t)-p(t))] \cos \left(2(\alpha+\eta(t))+\left(\lambda_{n}+\tilde{\lambda}_{n}\right) t\right) \mathrm{d} t+O\left(\frac{1}{n^{2}}\right) \\
= & \frac{1}{l_{n}^{j}} \int_{x_{n}^{j}}^{x_{n}^{j+1}}[(\tilde{q}(t)-q(t))-(\tilde{p}(t)-p(t))] \cos \left(2(\alpha+\eta(t))+\left(\lambda_{n}+\tilde{\lambda}_{n}\right) t\right) \mathrm{d} t+O\left(\frac{1}{n}\right) .
\end{aligned}
$$

Thus applying the result in [32], we derive with $j=j_{n}(x)$ that for $n \rightarrow \infty$

$$
[(\tilde{q}(x)-q(x))-(\tilde{p}(x)-p(x))] \cos \left(2(\alpha+\eta(x))+\left(\lambda_{n}+\tilde{\lambda}_{n}\right) x\right)=0,
$$

which means $q(x)-p(x)=\tilde{q}(x)-\tilde{p}(x)$, since the quantity $\cos \left(2(\alpha+\eta(x))+\left(\lambda_{n}+\tilde{\lambda}_{n}\right) x\right)$ cannot be identically zero for all $n \in \mathbb{Z}$ and $x \in[0,1]$. Hence we obtain from (3.9) that $p(x)=\tilde{p}(x)$, $q(x)=\tilde{q}(x)$ on $[0,1]$. The proof is complete. 
According to Lemma 3.1 one can choose sufficiently large $N_{1}$ such that for all $n>N_{1}$ there are exactly the first component $y_{1}\left(x, \lambda_{n}\right)$ of the eigenfunction $y\left(x, \lambda_{n}\right)$ and the first component $y_{1}\left(x, \lambda_{-n}\right)$ of the eigenfunction $y\left(x, \lambda_{-n}\right)$, corresponding to the eigenvalues $\lambda_{n}>0$ and $\lambda_{-n}<0$, respectively, of the operator $L$ that posses precisely $n$ nodes in the interval $(0,1)$. Analogous to the proof of [25], Theorem 6, the same assertion with the same $N_{1}$ is also valid for the operator $L_{a}$ of the form

$$
B y^{\prime}-Q(x) y=\lambda y, \quad 0 \leq a<x<1, \quad y_{1}(a)=V(y)=0,
$$

which can be formulated thus: for all $a \in[0,1)$ and $n>N_{1}$ there are exactly the first component $y_{1}\left(x, \lambda_{n, a}\right)$ of the eigenfunctions $y\left(x, \lambda_{n, a}\right)$ and the first component $y_{1}\left(x, \lambda_{-n, a}\right)$ of the eigenfunctions $y\left(x, \lambda_{-n, a}\right)$, corresponding to the eigenvalues $\lambda_{n, a}>0$ and $\lambda_{-n, a}<0$, respectively, for the operator $L_{a}$ possess precisely $n$ zeros in the interval $(a, 1)$.

Let us go on to the investigation of an incomplete inverse nodal problem when the nodal points are given only on a part of the interval.

Theorem 3.6 Fix $b<1 / 2$. If $X_{0} \cap(b, 1)=\tilde{X}_{0} \cap(b, 1)$ and $\eta(1)=\tilde{\eta}(1)$, then $L=\tilde{L}$. Thus, the specification of the nodes on any interval $(b, 1), b<1 / 2$, together with the mean values of $p+q$, uniquely determines the functions $p(x), q(x)$, and the coefficients $\alpha, \beta$ of the boundary conditions.

Proof By the same arguments in the proof of Theorem 3.4, it is easy to see that

$$
\beta=\tilde{\beta}, \quad p(x)=p(x), \quad q(x)=q(x) \quad \text { on }[b, 1] .
$$

Choose $N \geq N_{1}$ so that $x_{n}^{n-N_{1}-1}>b$ and $x_{-n}^{-n+N_{1}+2}>b$ for $n \geq N$. Fix $n \geq N$ and put $a:=x_{n}^{n-N_{1}-1}$. Consider the operator $L_{a}$, then for $n \geq N$ the component-function $y_{1}\left(x, \lambda_{n, a}\right)$ of the eigenfunction $y\left(x, \lambda_{n, a}\right)$ and the component-function $y_{1}\left(x, \lambda_{-n, a}\right)$ of the eigenfunction $y\left(x, \lambda_{-n, a}\right)$ for the operator $L_{a}$ both have precisely $n$ zeros in the interval $(a, 1)$, corresponding to the eigenvalues $\lambda_{n, a}>0$ and $\lambda_{-n, a}<0$, respectively. Moreover, these component-functions also satisfy the boundary conditions in (3.13) for the corresponding eigenvalues. Since the function $y_{1}\left(x, \lambda_{n}\right)$ has precisely $N_{1}$ zeros in the interval $(a, 1)$ and $V(y)=y_{1}\left(a, \lambda_{n}\right)=0$, thus we have $\lambda_{n}=\lambda_{N_{1}, a}$ or $\lambda_{n}=\lambda_{-N_{1}, a}$. Furthermore, (3.14) yields $\tilde{\lambda}_{n}=\lambda_{N_{1}, a}$ or $\tilde{\lambda}_{n}=\lambda_{-N_{1}, a}$. Since $\lambda_{n} \tilde{\lambda}_{n}>0$ and $\lambda_{N_{1}, a} \lambda_{-N_{1}, a}<0$, we get $\lambda_{n}=\tilde{\lambda}_{n}$. Analogously putting $a:=x_{-n}^{-n+N_{1}+2}$ we arrive at $\lambda_{-n}=\tilde{\lambda}_{-n}$. Thus, we have $\lambda_{n}=\tilde{\lambda}_{n}$ for $|n| \geq N$ and it remains to apply Theorem 2.3 to get $p(x)=\tilde{p}(x), q(x)=\tilde{q}(x)$ on $[0, b]$ and $\alpha=\tilde{\alpha}$. The proof is complete.

\footnotetext{
Competing interests

The authors declare that they have no competing interests.
}

Authors' contributions

The authors have made equal contributions to each part of this article. All the authors read and approved the final manuscript.

\section{Author details}

${ }^{1}$ College of Science, Xi'an Shiyou University, Xi'an, 710065, P.R. China. ${ }^{2}$ College of Mathematics and Information Science, Shaanxi Normal University, Xi'an, 710062, P.R. China. 


\section{Acknowledgements}

The research was supported in part by the NNSF (No. 11171198) and Fundamental Research Funds for the Centra Universities (No. GK 201304001) of China and Scientific Research Program Funded by Shaanxi Provincial Eduction Department (No. 2013JK0563).

\section{Received: 16 December 2014 Accepted: 17 March 2015 Published online: 18 June 2015}

\section{References}

1. Levitan, BM, Sargsjan, IS: Sturm-Liouville and Dirac Operators. Nauka, Moscow (1988) (in Russian); English transl., Kluwer Academic, Dordrecht (1991)

2. Freiling, G, Yurko, VA: Inverse Sturm-Liouville Problems and Their Applications. NOVA Science Publishers, New York (2001)

3. Levitan, BM: Inverse Sturm-Liouville Problems. VNU Science Press, Utrecht (1987)

4. Marchenko, VA: Sturm-Liouville Operators and Their Applications. Naukova Dumka, Kiev (1977); English transl., Birkhäuser, Basel (1986)

5. Yurko, VA: Inverse Spectral Problems for Differential Operators and Their Applications. Gordon \& Breach, Amsterdam (2000)

6. Kotelnikov, V: On the carrying capacity of the ether and wire in telecommunications. In: Material for the First All-Union Conference on Questions of Communications. Izd. Red. Upr. Svyazi RKKA, Moscow (1933) (in Russian)

7. Shannon, CE: Communications in the presence of noise. Proc. Inst. Radio Eng. 37, 10-21 (1949)

8. Whittaker, ET: On the functions which are represented by the expansion of the interpolation theory. Proc. R. Soc. Edinb., Sect. A 35, 181-194 (1915)

9. Tharwat, MM, Bhrawy, AH, Yildirim, A: Numerical computation of the eigenvalues of a discontinuous Dirac system using the sinc method with error analysis. Int. J. Comput. Math. 89, 2061-2080 (2012)

10. Tharwat, MM, Bhrawy, AH, Yildirim, A: Numerical computation of eigenvalues of discontinuous Sturm-Liouville problems with parameter dependent boundary conditions using sinc method. Numer. Algorithms 63, 27-48 (2013)

11. Tharwat, MM, Bhrawy, AH, Yildirim, A: Sampling of discontinuous Dirac systems. Numer. Funct. Anal. Optim. 34 323-348 (2013)

12. Tharwat, MM, Bhrawy, AH, Yildirim, A: Approximation of eigenvalues of discontinuous Sturm-Liouville problems with eigenparameter in all boundary conditions. Bound. Value Probl. 2013, 132 (2013)

13. Tharwat, MM, Bhrawy, AH: Computation of eigenvalues of discontinuous Dirac system using Hermite interpolation technique. Adv. Differ. Equ. 2012, 59 (2012)

14. Bhrawy, AH, Tharwat, MM, Al-Fhaid, A: Numerical algorithms for computing eigenvalues of discontinuous Dirac system using sinc-Gaussian method. Abstr. Appl. Anal. 2012, 925134 (2012)

15. Gasymov, MG, Dzhabiev, TT: Determination of a system of Dirac differential equations using two spectra. In: Proceedings of School-Seminar on the Spectral Theory of Operators and Representations of Group Theory, pp. 46-71. Élm, Baku (1975) (in Russian)

16. Gasymov, MG: Inverse problem of the scattering theory for Dirac system of order 2n. Tr. Mosk. Mat. Obŝ. 19, 41-112 (1968)

17. Horváth, M: On the inverse spectral theory of Schrödinger and Dirac operators. Trans. Am. Math. Soc. 353, 4155-4171 (2001)

18. Watson, BA: Inverse spectral problems for weighted Dirac systems. Inverse Probl. 15, 793-805 (1999)

19. Guo, Y, Wei, G: Inverse problems: dense nodal subset on an interior subinterval. J. Differ. Equ. 255, 2002-2017 (2013)

20. Law, CK, Shen, C-L, Yang, C-F: The inverse nodal problem on the smoothness of the potential function. Inverse Probl. $15,253-263(1999)$

21. Law, CK, Shen, C-L: On the well-posedness of the inverse nodal problem. Inverse Probl. 17, 1493-1512 (2001)

22. McLaughlin, JR: Inverse spectral theory using nodal points as data-a uniqueness result. J. Differ. Equ. 73, 354-362 (1988)

23. Yang, XF: A new inverse nodal problem. J. Differ. Equ. 169, 633-653 (2001)

24. Yang, C-F, Huang, Z-Y: Reconstruction of the Dirac operator from nodal data. Integral Equ. Oper. Theory 66, 539-551 (2010)

25. Buterin, SA, Shieh, C-T: Incomplete inverse spectral and nodal problems for differential pencils. Results Math. 62 , 167-179 (2012)

26. Gesztesy, F, Simon, B: Inverse spectral analysis with partial information on the potential: II. The case of discrete spectrum. Trans. Am. Math. Soc. 352, 2765-2787 (2000)

27. Shieh, C-T, Yurko, VA: Inverse nodal and inverse spectral problems for discontinuous boundary value problems. J. Math. Anal. Appl. 347, 266-272 (2008)

28. Horváth, M: Inverse spectral problems and closed exponential systems. Ann. Math. 162, 885-918 (2005)

29. Hochstadt, H, Lieberman, B: An inverse Sturm-Liouville problem with mixed given data. SIAM J. Appl. Math. 34, 676-680 (1978)

30. Malamud, MM: Uniqueness questions in inverse problems for systems of differential equations on a finite interval. Trans. Mosc. Math. Soc. 60, 173-224 (1999)

31. Levin, BY: Lectures on Entire Functions. Transl. Math. Monographs, vol. 150. Am. Math. Soc, Providence (1996)

32. Chen, YT, Cheng, YH, Law, CK, Tsay, J: $L^{1}$ Convergence of the reconstruction formula for the potential function. Proc Am. Math. Soc. 130, 2319-2324 (2002) 